\title{
A Clinical Challenge: Endocrine and Imaging Investigations of Adrenal Masses
}

\author{
Anders Sundin ${ }^{1}$, Elif Hindié ${ }^{2}$, Anca M. Avram ${ }^{3}$, Antoine Tabarin ${ }^{4}$, Karel Pacak $^{5}$, and David Taïeb ${ }^{6}$ \\ ${ }^{I}$ Section of Radiology \& Molecular Imaging, Department of Surgical Sciences, Uppsala University, Uppsala, Sweden; ${ }^{2}$ Department of \\ Nuclear Medicine, University Hospital of Bordeaux, Bordeaux, France; ${ }^{3}$ Department of Radiology, University of Michigan, Ann Arbor, \\ Michigan; ${ }^{4}$ Department of Endocrinology, University Hospital of Bordeaux, Bordeaux, France; ${ }^{5}$ Section on Medical Neuroendocrinology, \\ Eunice Kennedy Shriver National Institute of Child Health \& Human Development (NICHD), National Institutes of Health, Bethesda, \\ Maryland; and ${ }^{6}$ Department of Nuclear Medicine, La Timone University Hospital, CERIMED, Aix-Marseille University, Marseille, France
}

Incidentalomas are reported in $3 \%-4 \%$ of patients who undergo abdominal anatomic imaging, making adrenal mass evaluation a common occurrence. An adrenal mass can be caused by a variety of pathologies, such as benign cortical and medullary tumors, malignant tumors (primary or secondary), cysts, hyperplasia, hemorrhage, or more rarely infection/inflammation processes. Functioning tumors usually have increased hormonal production but they are less common. Regardless of their functional status, some tumors have the potential to behave aggressively. Anatomic and functional imaging together with biologic evaluation play a vital role in adrenal pathology subtyping. Most patients are initially evaluated by CT or MRI, which allows for tumor characterization (to a certain extent) and can rule out malignant behavior based on the absence of tumor growth during longitudinal follow-up. In the remaining patients for whom CT or MRI fail to characterize the pathogenesis of adrenal tumors, the use of specialized molecular imaging techniques should be performed after hormonal screening. This review emphasizes well-established and emerging nuclear medicine imaging modalities and describes their use across various clinical scenarios.

Key Words: adrenal; ${ }^{18}$ F-FDG; PET/CT; CT; incidentaloma; adrenocortical carcinoma

Abbreviations: RET: rearranged during transfection proto-oncogene; $\mathrm{SDH}$ : succinate dehydrogenase; SDHA, -B, -C, -D: succinate dehydrogenase subunits A, B, C, D; SDHx: succinate dehydrogenase subunits; NF1: neurofibromin 1; MAX: myc-associated factor X; TMEM127: transmembrane protein 127; CYP: cytochrome P450 superfamily; ARMC5: armadillo repeat containing 5; PRKAR1A: protein kinase A regulatory subunit type IA

J Nucl Med 2021; 62:26S-33S

DOI: 10.2967/jnumed.120.246066

\begin{abstract}
A drenal incidentalomas are adrenal masses greater than $1 \mathrm{~cm}$ discovered on imaging that is performed for other indications (i.e., reasons other than adrenal disease) (1). Due to the extensive and continuous increase of abdominal imaging, the management of adrenal incidentalomas has become a growing clinical concern. Understanding the epidemiology of incidentalomas, which are benign lesions in most cases (2), has resulted in simplifying and streamlining of the imaging procedures and follow-up routines for adrenal incidentalomas. Biochemical testing of secretory function (screening
\end{abstract}

Received Jun. 22, 2020; revision accepted Oct. 26, 2020.

For correspondence or reprints, contact David Taïeb (david.taieb@ap-hm.fr). COPYRIGHT (C) 2021 by the Society of Nuclear Medicine and Molecular Imaging. for mineralocorticoid, glucocorticoid, and catecholamine secretion) is necessary to distinguish functional versus nonfunctional adrenal lesions. Dedicated imaging is required to differentiate benign and malignant adrenal lesions.

\section{ADRENAL INCIDENTALOMAS AND PATIENTS PRESENTING WITH ADRENAL DISEASE}

Radiologic characterization of many adrenal incidentalomas, including hematomas, cysts, and myelolipomas, can be executed by $\mathrm{CT}$ and MRI. Most (70\%) benign adrenocortical adenomas show low attenuation on precontrast CT $(\leq 10$ Hounsfield units [HU]) (3) and chemical shift on MRI (4) due to their high content of cytoplasmatic fat (5). Malignant tumors can also be excluded on the basis of lesion stability on follow-up imaging at least 6-12 mo apart. Nonfunctioning adrenal incidentalomas $(<4 \mathrm{~cm}$ in size $)$ that are not fully characterized on CT/MRI but demonstrate lack of growth during longitudinal follow-up ( $\geq 6-12 \mathrm{mo}$ ) are considered benign and do not need further imaging. Lesions $\geq 4 \mathrm{~cm}$ are usually discussed with a multidisciplinary board to determine individualized patient management. Patients with functioning or uncharacterized adrenal incidentalomas and patients with symptoms or biochemistry indicating adrenal pathology need further molecular imaging studies to provide an accurate diagnosis.

\section{DIVERSITY OF IMAGING MODALITIES}

Adrenal imaging is characterized by a wide variety of modalities. For the large majority of patients, CT or MRI is sufficient to characterize adrenal lesions, to exclude malignant disease (serial followup), and for imaging of patients with symptoms or biochemistry indicating adrenal disease. In selected patients, functional methods can provide additional diagnostic information, important for the clinical management. Several radiopharmaceuticals for nuclear medicine imaging have been developed to visualize the following:

1. The adrenal cortex via different target structures, using a cholesterol analog such as ${ }^{131}$ I- $6 \beta$-iodomethyl-19-norcholesterol (named ${ }^{131} \mathrm{I}-\mathrm{NP}-59$ by researchers who have synthetized this compound), which is incorporated in low-density lipoproteins or CYP11B inhibitors $\left({ }^{11} \mathrm{C}\right.$-metomidate, ${ }^{123}$ I-iodometomidate). The final steps of production of glucocorticoids and mineralocorticoids are catalyzed by the CYP11B subfamily regrouping 2 mitochondrial cytochromes P450, CYP11B1 (11 $\beta$-hydroxylase) expressed across all adrenocortical zones and CYP11B2 restricted to aldosterone-producing cells (aldosterone synthase). 
2. The adrenal medulla by targeting the molecular machinery underlying the successive steps of uptake and catecholamines synthesis in chromaffin cells: ${ }^{123} \mathrm{I} /\left({ }^{131} \mathrm{I}-\mathrm{MIBG},{ }^{18} \mathrm{~F}-\mathrm{FDA},{ }^{11} \mathrm{C}-\right.$ hydroxyephedrine, which enter into the cells via the norepinephrine transporter, and ${ }^{18} \mathrm{~F}$-FDOPA, which enters via the L-type amino acid transporter family.

3. Increased glucose metabolism with ${ }^{18} \mathrm{~F}-\mathrm{FDG}$, which reflects an emerging hallmark of cancer cells called metabolic reprogramming (e.g., distinct metabolic phenotypes to fuel proliferation and progression).

4. Overexpression of some receptors such as somatostatin receptors (SSRs) that bind somatostatin analogs and ${ }^{68} \mathrm{Ga}-\mathrm{SSA}$ for SSR or $\mathrm{C}-\mathrm{X}-\mathrm{C}$ chemokine receptor type 4 (CXCR4) that respond to CXCL12 (SDF-1) analogs and trigger chemotaxis of lymphocytes and bind ${ }^{68} \mathrm{Ga}$-pentixafor for CXCR4 (Fig. 1).

Technical aspects are not addressed in this review; rather, the role of nuclear imaging modalities across a variety of clinical settings, tailored toward the individual patient and the clinical scenario, is emphasized.

\section{CURRENT KEY STEPS IN ASSESSING A PATIENT WITH AN ADRENAL MASS}

The following steps can be proposed for assessing a patient with an adrenal mass (Fig. 2; Table 1).

\section{First Step: Radiologic Analysis with Clinical and Biochemical Evaluation}

Exclude Potential Pitfalls That Could Disguise as an Adrenal Mass. There are several examples of pseudoadrenal tumors related to the encroaching of adjacent normal structures (e.g., diaphragmatic crus, gastric diverticulum) or adjacent disease processes (renal, pancreas, soft tissue) (6). These can usually be distinguished from true

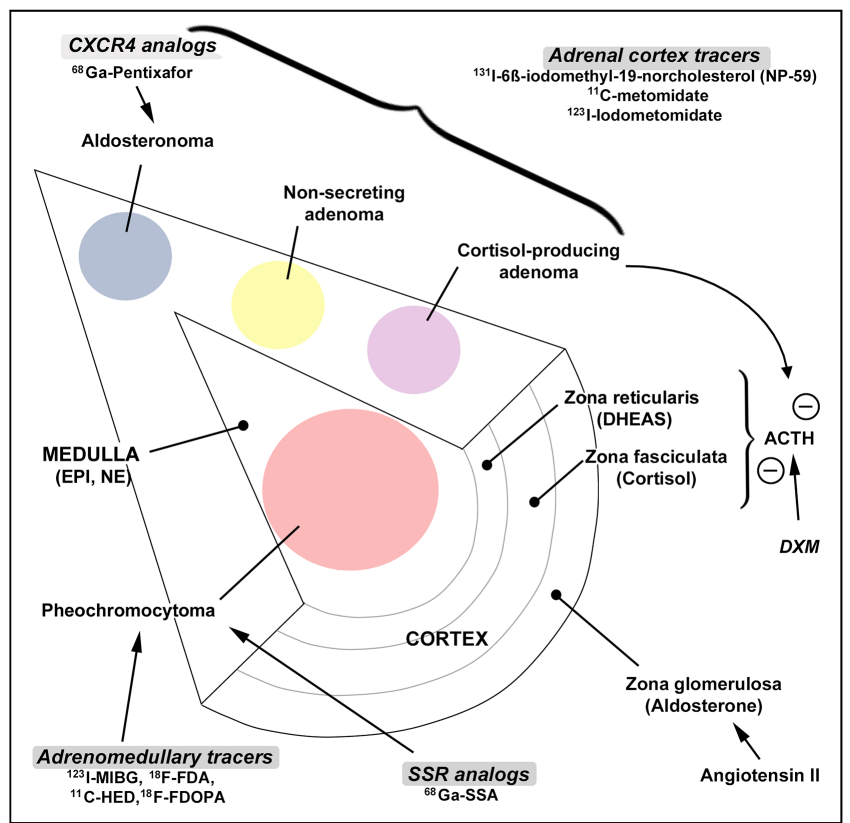

FIGURE 1. Schematic display of radiopharmaceuticals for adrenal cortex and medulla tumors imaging. DXM (used for premedication before NP-59 or ${ }^{11} \mathrm{C}$-metomidate imaging in primary hyperaldosteronism) = dexamethasone; EPI = epinephrine; NE: norepinephrine; SSR = somatostatin receptors.

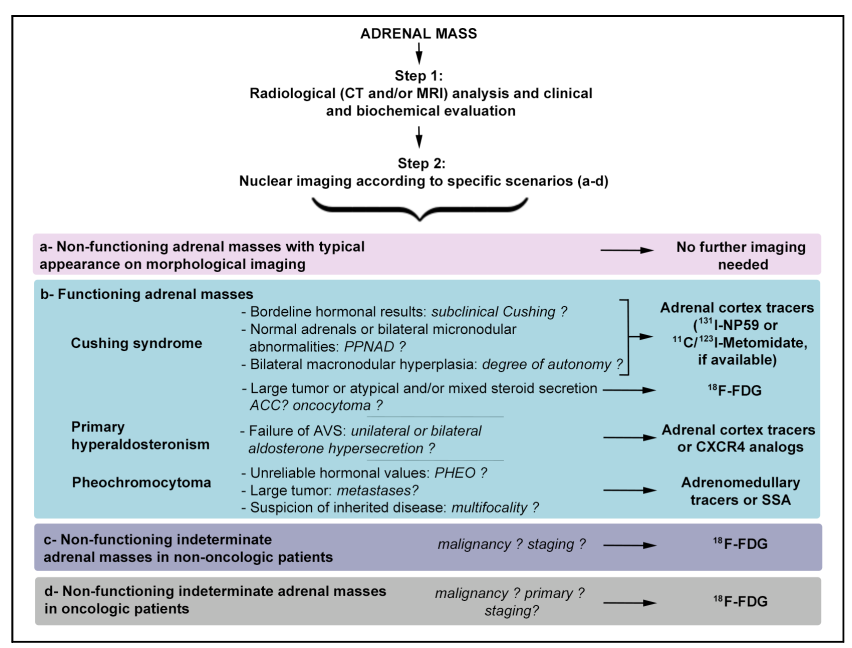

FIGURE 2. Stepwise algorithm of nuclear imaging in the evaluation of adrenal masses.

adrenal lesions. Morphologic image analysis in the transversal, coronal, and sagittal planes is often helpful. However, large masses arising from the soft tissues of the retroperitoneum or the adjacent organs may disguise as an adrenal mass.

Assess Benign and Malignant Characteristics on Morphologic Imaging. Once an adrenal tumor has been established, its benign or malignant nature needs to be assessed. In most patients, tumor characterization is managed by conventional radiologic imaging (i.e., CT or MRI) $(7,8)$. Sharp delineation, rounded harmonic shape, and homogeneous internal structure are usually considered morphologically benign characteristics. Infiltrative margins, lobulated irregular shape and heterogeneous internal structure may indicate that the tumor is malignant. Additionally, large tumors are more likely to be malignant than small ones. Since malignant tumors grow at a fast rate, unchanged tumor size in comparison to a previous CT/MRI ( $>6 \mathrm{mo}$ ) will strongly indicate that the tumor is benign (9).

Certain types of adrenal tumors can be diagnosed based on their typical CT/MRI appearance, such as presence of water and absence of contrast-enhancement in cysts or hematopoietic tissue and macroscopic fat in myelolipomas. An adrenocortical adenoma is the most common adrenal tumor and is characterized by benign morphologic characteristics on CT/MRI and high levels of cytoplasmatic fat. Because the attenuation of the parenchymal organs on precontrast (native) $\mathrm{CT}$ ranges approximately 30-70 HUs, the presence of microscopic fat (-100 $\mathrm{HU}$ ) in the cytoplasm of an adrenocortical adenoma will lower the attenuation. Therefore, tumor attenuation $\leq 10 \mathrm{HU}$ in a morphologically benign tumor (according to appearance on CT/MRI) can be characterized as a lipid-rich adrenal adenoma. A study has shown that it is possible to safely extend this threshold to $20 \mathrm{HU}$ assuming that the tumor is homogeneous and $<30 \mathrm{~mm}$ in size (10). Microscopic fat in adrenocortical adenomas can also be shown on in-phase and out-ofphase MRI sequences. Patients with larger tumors $>4 \mathrm{~cm}$ (except those with a typical cyst or myelolipoma) are generally discussed with a multidisciplinary team. Approximately $30 \%$ of adrenocortical adenomas do not have enough cytoplasmic fat for proper characterization on CT/MRI and are thus categorized as lipid-poor adrenal adenomas.

The use of triple-phase adrenal CT with calculation of contrast medium washout is still widely used for characterization of indeterminate adrenal lesions, although with varying practice in different countries. A diagnostic algorithm for managing incidental adrenal 
Etiology

\begin{tabular}{|c|c|}
\hline Adrenocortical adenoma & Usually normal, possible Cushing syndrome, primary hyperaldosteronism or hyperandrogenism \\
\hline Pheochromocytoma & $\begin{array}{l}\text { Hypertension, paroxysms (palpitations, pallor, tremor, headache, diaphoresis), possible family } \\
\text { history of hereditary disease or syndromic, manifestations, increased urinary or plasma } \\
\text { metanephrines, May be asymptomatic }\end{array}$ \\
\hline Myelolipoma & Usually normal, possible chronic flank pain and abdominal discomfort \\
\hline Adrenal hemorrhage & $\begin{array}{l}\text { Possible history of trauma, thrombophilia (including antiphospholipid, antibody syndrome), } \\
\text { underlying adrenal tumor, adrenal venous sampling, possible acute adrenal insufficiency (if } \\
\text { bilateral) }\end{array}$ \\
\hline ACC & $\begin{array}{l}\text { Severe Cushing syndrome or hyperandrogenism (frequent mixed secretion), rare } \\
\text { hyperaldosteronism or estrogen secretion, abdominal or flanck pain }\end{array}$ \\
\hline Ganglioneuroma & Usually normal, possible catecholamines secretion in composite tumors \\
\hline Adrenal cyst & Usually normal, possible chronic flank pain and abdominal discomfort \\
\hline Adrenal metastasis & $\begin{array}{l}\text { History of an extraadrenal cancer, cancer-associated signs and markers, possible adrenal } \\
\text { insufficiency (if bilateral) }\end{array}$ \\
\hline Primary adrenal lymphoma & $\begin{array}{l}\text { Worsening general state, abdominal or flank pain, increased LDH, } \beta 2 \text { microglobulin, CRP or } \\
\text { ferritinemia levels, possible adrenal insufficiency (if bilateral) }\end{array}$ \\
\hline
\end{tabular}

$\mathrm{LDH}=$ serum lactate dehydrogenase; $\mathrm{CRP}=$ serum C-reactive protein.

masses was updated in 2017 by the American College of Radiology (ACR Incidental Findings Committee) (8). By contrast, the guidelines by the European Society of Endocrinology and European Network for the Study of Adrenal Tumors do not use this approach (9), which was based on the results of a systematic review and metaanalysis (2). Further, adrenal CT washout has been shown to be falsepositive in pheochromocytomas $(2,9,11)$.

Adrenal bleeding (especially with a history of acute trauma) will usually appear as a typical hematoma with unenhanced attenuation value of 55-90 HUs and will be diagnosed as such on noncontrast (native) CT imaging. An adrenal hematoma will typically decrease in size on follow-up imaging, with a gradual decrease in attenuation value. A spontaneous bleed in an adrenal gland is usually silent and therefore not generally diagnosed on CT/MRI. On late stages of CT/ MRI, the remnants of an adrenal hematoma (especially when composed of fibrosis) may appear as an adrenal tumor and escape characterization.

Adrenocortical carcinomas (ACC) are rare and usually appear on $\mathrm{CT} / \mathrm{MRI}$ as a large, irregular, and heterogeneous tumor with predominantly peripheral contrast-enhancement due to central necrosis. Pheochromocytomas typically have heterogeneous contrast-enhancement, but can deviate from this pattern and show a wide range of appearances. A large pheochromocytoma may sometimes mimic an ACC. In patients with adrenal metastases, the cancer type is generally known, and these patients usually undergo restaging. Notably, adrenal tumors in patients with a cancer history are benign in three quarters of the time (12). If a tumor biopsy is indicated, clinicians must ensure that a biochemically active pheochromocytoma has been excluded to avoid complications. Rare etiologies such as unilateral adrenal lymphomas, sarcomas, and large, nonsecreting ACCs represent a true diagnostic challenge and can only be solved by a biopsy.

Determine if the Mass Is Hormonally Active. Although most adrenal masses are nonfunctioning adenomas, a careful investigation including family history, primary neoplasm history, and physical examination must be performed. Hormonal evaluation can reveal clinically unsuspected, functioning adrenal tumors. The most common hormones that can be oversecreted are aldosterone and cortisol from the cortex and epinephrine and norepinephrine from the medulla. The first step should include an overnight low-dose dexamethasone suppression test, the measurement of fractionated plasma metanephrines or 24-h urinary metanephrines, serum potassium, and, in hypertensive patients, upright plasma aldosterone/plasma renin activity ratio. Dehydroepiandrosterone (DHEA/DHEA-S) measurements may show evidence of adrenal androgen excess. Positive results should be followed by specific hormonal evaluations for a definitive diagnosis (9).

\section{Second Step: Distinguish the Clinical Scenarios}

Nonfunctioning Adrenal Masses with a Typical Appearance: Functional Imaging Is Not Recommended. These tumors include adrenocortical adenomas, myelolipomas, and cysts (the characterization has been described above). In addition to lipid-poor adrenocortical adenomas, other adrenal tumors characterized as benign include ganglioneuroma, lymphangioma, benign oncocytoma, and schwannoma, based on size stability on CT/MRI at least 6-12 mo apart. These are usually not diagnosed histopathologically unless the patient subsequently undergoes surgical resection or biopsy.

Functioning Adrenal Masses: Functional Imaging May Be Appropriate (Cushing Syndrome, Primary Hyperaldosteronism [PHA], and Pheochromocytoma). Adrenal Cushing syndrome (also called ACTH-independent) is mainly caused by benign or malignant unilateral adrenocortical tumors. In rare cases, adrenal Cushing syndrome is caused by primary bilateral macronodular adrenal hyperplasia (PBMAH) or primary micronodular bilateral adrenal hyperplasia, mainly related to primary pigmented nodular adrenocortical disease (PPNAD) or less frequently related to isolated 
micronodular adrenocortical disease. PBMAH has been attributed to germline mutations in the Armadillo repeat containing 5 (ARMC5) gene in about $50 \%$ of cases. ARMC5 is located on chromosome 16 (16p11.2) and acts as an adrenal gland tumor suppressor gene.

PPNAD is often familial and associated with multiple endocrine neoplasia syndrome and the Carney complex and caused by germline PRKAR1A mutations in up to 70\%. PRKAR1A (chromosome 17q22-24) encodes the protein kinase A (PKA) regulatory subunit type IA, an important regulator of cAMP signaling in most cells. PPNAD is usually diagnosed before the age of $30 \mathrm{y}$, with $50 \%$ of patients being younger than $15 \mathrm{y}$ at diagnosis (13).

Several clinical scenarios can be distinguished:

1. Presence of a typical adrenocortical adenoma: for these patients, there is no need for nuclear medicine imaging. In the presence of borderline hormonal results, adrenocortical imaging can be performed to confirm autonomous secretion of cortisol by the adenoma. This was historically demonstrated with NP-59 scintigraphy that typically shows a unilateral tumor uptake. Adenoma cortisol secretion suppresses pituitary ACTH production and tracer uptake by the contralateral adrenal gland. In some centers ${ }^{11} \mathrm{C}$-metomidate-PET/CT is available for disease characterization (Fig. 1).

2. Presence of a large heterogeneous mass: the disease is most likely related to an ACC and the patient should undergo ${ }^{18} \mathrm{~F}-\mathrm{FDG}$ PET/ CT for initial staging. The main differential diagnosis is adrenal oncocytoma (benign or malignant); however, differential diagnosis is almost impossible when relying solely on imaging. Of note, oncocytomas are characterized by very high ${ }^{18}$ F-FDG uptake; however, this can also be observed in ACCs that contain a substantial oncocytic component. If available, ${ }^{11} \mathrm{C}$-metomidate$\mathrm{PET} / \mathrm{CT}$ also allows a noninvasive characterization and staging of ACC (14). ${ }^{11} \mathrm{C}$-metomidate binds to the $11 \beta$-hydroxylase enzyme, which is involved in the last step of the synthesis of cortisol and aldosterone.

3. Presence of unilateral or bilateral micronodules or normal adrenals: the diagnosis is most likely related to PPNAD. Physicians should be aware that radiologic imaging can be misleading in some cases of PPNAD with unilateral abnormality (micronodules with or without coexistence of macronodules). In PPNAD, adrenal cortex functional imaging using NP-59 typically shows a bilateral adrenal uptake with possible asymmetrical uptake in patients with macronodules (15).

4. Presence of PBMAH: in this situation, adrenal cortex imaging may help when choosing the most appropriate surgical approach. Unilateral adrenalectomy can offer prolonged remission (only $20 \%$ recurrence) and is associated with about $40 \%$ of incomplete and transient secondary adrenal insufficiency cases. This postoperative adrenal insufficiency illustrates the low production yield of cortisol per milligram of tissue since it is produced by a subpopulation of steroidogenic cells in the hyperplastic adrenals (16). One hundred percent remission may be achieved with bilateral adrenalectomy, but this induces definitive adrenal insufficiency with lifelong need of steroid replacement and risk for adrenal crisis. The best criteria for choosing an adrenalectomy are still unknown. Adrenal vein catheterization to compare cortisol concentration between the right and left adrenal effluents has not gained acceptance to date. Adrenal cortex imaging with quantitative analysis could play a role for guiding surgery, especially when masses have similar volumes. In all these situations, radiopharmaceuticals that target the CYP11B enzyme family are of interest and have the potential to provide clinical benefits.

Primary hyperaldosteronism (PHA) represents the most common form of secondary hypertension, with a prevalence of up to $12 \%$ of hypertensive patients. The most frequent subforms are idiopathic hyperaldosteronism or idiopathic adrenal hyperplasia and aldosterone-producing adenoma. Rare causes include unilateral hyperplasia, ACC, and familial hyperaldosteronism. Studies conducted in the last 8 y have identified somatic driver mutations in a substantial percentage of aldosterone-producing adenoma. Differentiation between aldosterone-producing adenoma and bilateral hyperplasia is essential when considering further treatments that rely on lifelong medications (mineralocorticoid receptors antagonists or potassium-sparing diuretics) or adrenalectomy. This can be challenging because nonfunctioning adenoma can coexist with aldosterone-producing adenoma or bilateral hyperplasia, and a unilateral nonfunctioning adenoma can be incidentally detected on CT/ MRI in bilateral adrenal hyperplasia. The use of conventional imaging alone in the etiologic diagnosis of PHA could lead to inappropriate therapeutic strategies in up to $40 \%$ of cases. Adrenal venous sampling (AVS) is the gold standard for subtyping PHA and surgical teams that use AVS achieve better results, as assessed by postoperative decreases in blood pressure (17). This beneficial effect on outcome has not been reported in the SPARTACUS trial (Subtyping Primary Aldosteronism: a Randomized Trial Comparing Adrenal Vein Sampling and CT Scan, NCT01096654), although this trial was somewhat hampered by some cohort and endpoint bias $(18,19)$. It is noteworthy, however, that not all patients need to undergo AVS. Younger patients (age $<35$ y) with spontaneous hypokalemia, marked aldosterone excess, and unilateral adrenal lesions who have radiologic features consistent with a cortical adenoma on adrenal CT scan may not need AVS before proceeding to unilateral adrenalectomy. Nuclear medicine imaging is classically positioned after unsuccessful AVS localization, which is not uncommon. AVS is technically difficult, especially cannulating the right adrenal vein from the inferior vena cava (attested by low selectivity index). Even with correct placement of the catheter in the right adrenal vein, the sampling technique is critical, and simultaneously drawing blood from the inferior vena cava should be avoided. Furthermore, there are some discrepancies regarding performances across centers, interpretation of the results, and technical approaches
FIGURE 3. Aldosterone-producing adenoma in a patient with primary hyperaldosteronism. A 55-y-old man with primary aldosteronism and inconclusive adrenal venous sampling. ${ }^{11} \mathrm{C}$-metomidate PET/CT after $5 \mathrm{~d}$ of $1 \mathrm{mg} \times 3$ dexamethasone suppression shows high focal tracer uptake $\left(\mathrm{SUV}_{\max }=24.3\right)$ in 8-mm nodule in the lateral aspect of the left adrenal gland consistent with an aldosterone-producing adenoma (arrows). Uptake in the normal contralateral adrenal is low (arrowhead). Physiologic uptake is seen in gastric juice. (A) Axial PET. (B) Axial PET/CT fusion. (C) Coronal PET/CT fusion. 
(e.g., using or not using cosyntropin stimulation). The role of nuclear medicine will be greatly dependent on the development of PET radiopharmaceuticals. Except for ${ }^{68} \mathrm{Ga}$-pentixafor PET/CT, all nuclear imaging investigations require premedication with dexamethasone to achieve ACTH suppression, with the goal of inhibiting the cortisol-producing cortical tissue that could mask the underlying disease. Although ${ }^{131}$ I-NP-59 is not widely available any longer, it may be useful in evaluating patients with primary hyperaldosteronism for adrenalectomy when AVS is inconclusive. ${ }^{131}$ I-NP-59 scintigraphy, however, has several practical drawbacks such as limited spatial resolution and relatively high radiation dose exposure (effective dose estimated to $1.5 \mathrm{mSv} / \mathrm{MBq}$ ). Along with this, patients must withdraw from interfering medications that may provoke (together with dexamethasone) a hypertension flair. Use of SPECT/CT with NP-59 scintigraphy allows precise localization of the functional activity to anatomic structures improving diagnostic interpretation (20).

To circumvent the main shortcomings of ${ }^{131}$ I-NP-59 imaging, several tracers that target the CYP11B enzyme family and chemokine-receptor CXCR4 ( ${ }^{68} \mathrm{Ga}$-pentixafor) have been developed (Fig. 1).

For patients with PHA, ${ }^{11} \mathrm{C}$-metomidate-PET/CT has been used for visualization of aldosterone-producing adenoma (Fig. 3) (21). In addition to its limited availability, the clinical use of ${ }^{11} \mathrm{C}$-metomidate is also limited by its focal uptake in nonfunctioning adenoma, which also requires suppression of ACTH for $5 \mathrm{~d}$ of dexamethasone administration before PET/CT imaging. The diagnosis of aldosterone-producing adenoma relies on the higher tumor $\mathrm{SUV}_{\max }$ compared with the contralateral gland (22) and can be less conclusive in small adenoma. The results from an ongoing British trial MATCH (NCT02945904) comparing ${ }^{11} \mathrm{C}$-metomidate and AVS for lateralization in PHA with correlation to surgery and histopathology are still pending.

The use of ${ }^{123}$ I-iodometomidate for SPECT is limited by the reduced spatial resolution and the need to premedicate patients with dexamethazone (23). ${ }^{68} \mathrm{Ga}$-pentixafor is likely the most promising PET radiotracer. Interestingly, CXCR4 expression is almost

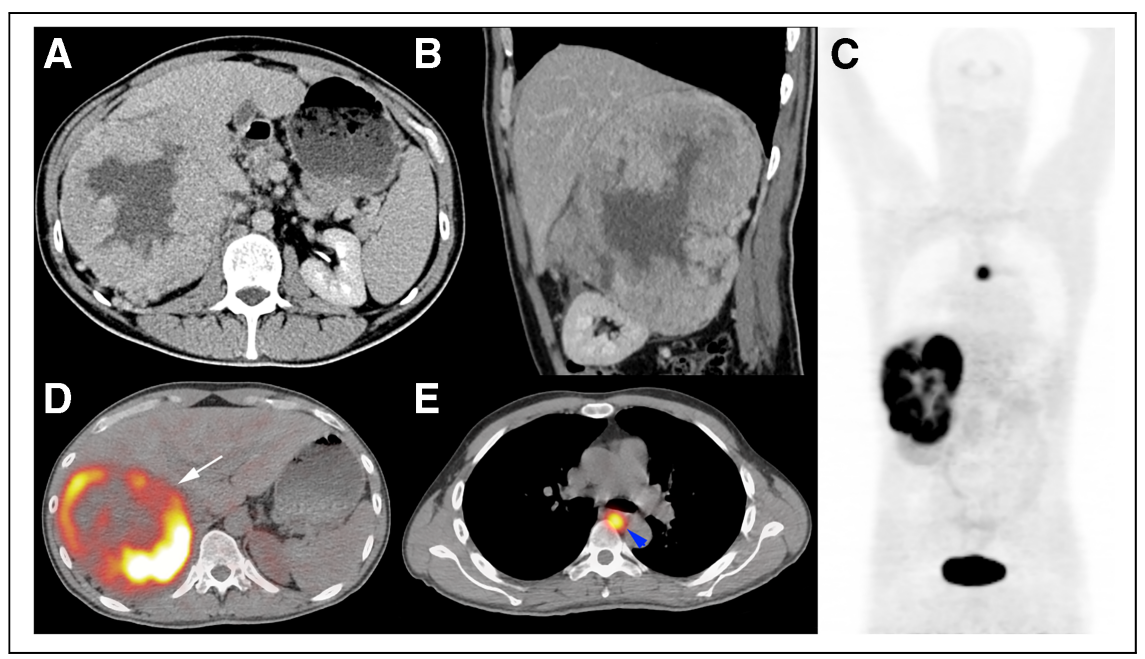

FIGURE 4. Large pheochromocytoma (sporadic) with mediastinal lymph node metastasis. (A and B) Large heterogeneous mass on CT ([A] axial plane, $[\mathrm{B}]$ sagittal plane). (C-E) Marked tumor avidity for ${ }^{18} \mathrm{~F}-$ FDOPA (arrow) with visualization of subcarinal posterior mediastinal lymph node (arrowhead) and not a second paraganglioma based on pathologic analysis ([C] maximum-intensity projection [MIP], [D and E] axial PET/CT fusion). negligible in nonfunctioning adenomas on immunohistochemical studies (24). In a prospective study that included 36 patients with PHA, ${ }^{68}$ Ga-pentixafor was effective in distinguishing aldosteroneproducing adenoma from nonfunctioning adenoma and hyperplasia (25). On immunohistochemistry analysis, 24 of 25 aldosteroneproducing adenoma showed an intense CXCR4 expression, whereas the remaining lesions (4 hyperplasia and 5 nonfunctioning adenoma) did not express (5/11) or slightly expressed (6/11) CXCR4. Considering the correlation between CXCR4 expression and tracer uptake, the use of quantitative indices increases the diagnostic value of ${ }^{68} \mathrm{Ga}$-pentixafor PET/CT. In conclusion, targeted molecular imaging (NP-59, ligands of the CYP11B enzyme family or CXCR4) could be a reliable tool in patients with PHA in the absence of a clear-cut AVS result and potential indication for surgery. It is expected by clinicians and nuclear physicians that these emerging modalities could be positioned before AVS in the near future. Development of highly selective CYP11B2 ligands might also be useful for nuclear imaging in PHA but is challenging due to the high homology of CYP11B1 and CYP11B2. Selective radiolabeled inhibitors of CYP11B2 have so far been evaluated in preclinical studies.

Pheochromocytoma (PHEO) arises from the chromaffin cells of the adrenal medulla and typically produce both norepinephrine and epinephrine. Metastatic PHEO are rare and usually large, although tumor size is a less reliable predictor for malignancy compared with adrenocortical tumors. PHEOs are usually sporadic but they can also be related to inherited genetic mutations such as RET (MEN2A and MEN2B), von Hippel-Lindau (VHL), SDHx, $N F-1, M A X$, and TMEM127. In apparently sporadic cases or cases with negative genetic testing, functional imaging should be prioritized in selected cases to confirm the diagnosis in rare situations (e.g., typical imaging appearance with normal metanephrines, or unreliable laboratory values due to acute critical illness) or exclude malignancy in large PHEOs. In suspected hereditary cases (syndromic manifestations, family history, multifocality, young age and exclusive norepinephrine secretion), functional imaging is recommended to screen for potential multifocality and malignancy, especially in $S D H B$ cases. In sporadic cases, either ${ }^{18} \mathrm{~F}$-FDOPA or ${ }^{123} \mathrm{I}-\mathrm{MIBG}$ can be used as first choice tracers (Fig. 4). RET-, NF1-, MAX-, and TMEM127-related tumors, which are almost always confined to the adrenal medulla and are well-differentiated, are better evaluated with ${ }^{18} \mathrm{~F}$-FDOPA PET/CT (26). The low uptake of ${ }^{18} \mathrm{~F}$ FDOPA by healthy adrenals facilitates the detection of subcentimeter PHEO (microPHEO) that can coexist within the same or contralateral adrenal gland. For SDHx cases, the use of ${ }^{68} \mathrm{Ga}$-SSA $\mathrm{PET} / \mathrm{CT}$ imaging is recommended as a first choice despite uptake by healthy adrenals (27-29). ${ }^{11} \mathrm{C}$-hydroxyephedrine is a norepinephrine analog and has been used as a PET tracer for the diagnosis of PHEO (30); however, its availability is restricted to merely a few centers. In a recent study in patients with borderline elevated metanephrines or symptoms consistent with PHEO, ${ }^{11} \mathrm{C}$-hydroxyephedrine PET/CT allowed for the diagnosis or exclusion of 


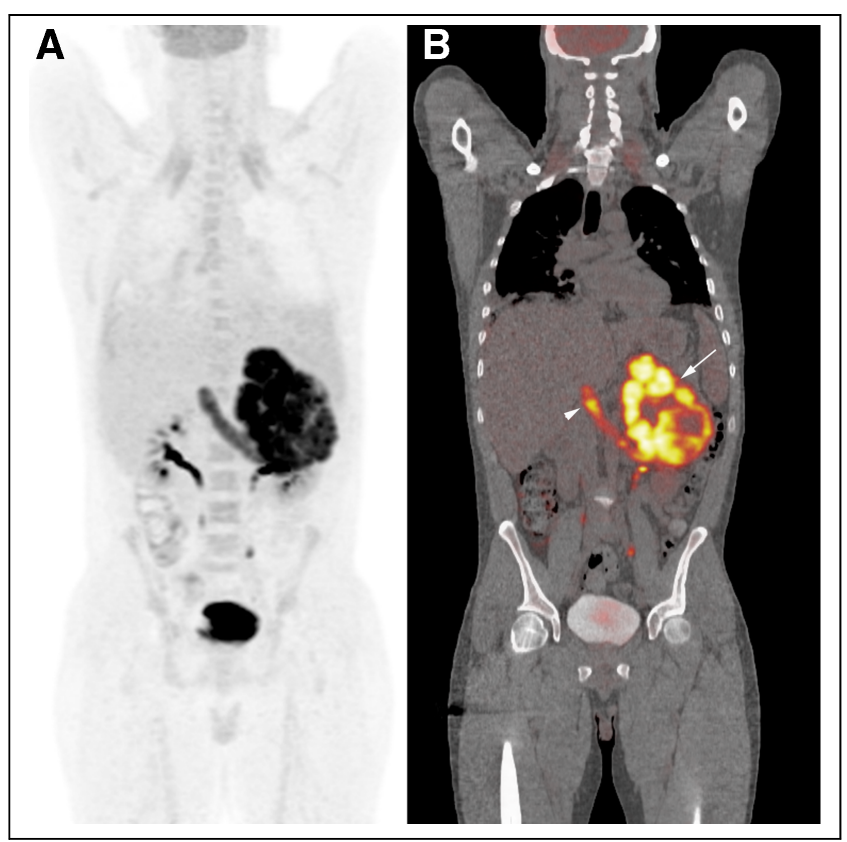

FIGURE 5. ${ }^{18} \mathrm{~F}-\mathrm{FDG}$ PET/CT in ACC with extension into inferior vena cava. ( $A$ and B) Left ACC (arrow) with tumor thrombus extending via left adrenal and renal veins into inferior vena cava (arrowhead) $([\mathrm{A}]$ maximum-intensity projection [MIP], [B] coronal PET/CT fusion).

diagnosis for PHEO. Furthermore, ${ }^{11} \mathrm{C}$-hydroxyephedrine PET/CT has also been used for the staging of malignant $\mathrm{PHEO}$, postoperative surveillance, and detection of recurrent disease (31).

The clinical decision making in the selection of metastatic PHEO patients for appropriate targeted radionuclide therapy (conventional/novel high-specific-activity ${ }^{131}$ I-MIBG therapy or ${ }^{177} \mathrm{Lu}-$ DOTATATE) should be personalized to the imaging of phenotypes on ${ }^{123} \mathrm{I}-\mathrm{MIBG}$ and ${ }^{68} \mathrm{Ga}$-SSA scans.

Nonfunctioning Indeterminate Adrenal Masses in Nononcologic Patients: Functional Imaging May Be Appropriate. Several studies have shown that assessment of tumor metabolism by ${ }^{18} \mathrm{~F}-\mathrm{FDG}$ PET/ CT can help characterize large or indeterminate masses. This is important to achieve early diagnosis of malignant tumors and avoid unnecessary resection of benign lesions. When surgery is indicated, there is also a wide range of surgical techniques that may be used, including cortical-sparing adrenalectomy, adrenalectomy, adrenalectomy with removal of the periadrenal fat, and extensive resection to obtain as much as possible negative resection margins and with laparoscopic or open surgery. Overall, management of adrenal masses can be influenced by ${ }^{18} \mathrm{~F}-\mathrm{FDG}$ PET/CT findings. Although ${ }^{18}$ F-FDG PET/CT can be helpful to differentiate patients with malignant tumors from those with benign lesions, there is an uncertainty in a gray zone when ${ }^{18} \mathrm{~F}$-FDG uptake is neither very low nor very high (32). ACC has been consistently demonstrated to display high metabolic activity on ${ }^{18}$ F-FDG PET imaging. Because of the poor prognosis, marked by a high incidence of disseminated disease at presentation and high recurrence rate after initial complete resection, patients undergo repeated imaging evaluation for staging and restaging of the disease. ${ }^{18} \mathrm{~F}$-FDG PET/CT plays an important role in both the initial ACC staging and in the subsequent follow-up staging.

The use of tumor-to-liver uptake $\mathrm{SUV}_{\max }$ ratio was found to be more accurate than visual analysis in the distinction between benign and malignant tumors, with an optimal threshold varying across studies. In a prospective study, we have previously shown that a ratio
$>1.5$ was associated with malignancy, with sensitivity, specificity, positive predictive value, negative predictive value, and accuracy of $86.7 \%, 86.1 \%, 56.5,96.9$, and $86.2 \%$, respectively (33). Although this cutoff value is useful, it is well known that some adrenocortical cancers and large retroperitoneal sarcomas may exhibit lower uptake values. Several imaging patterns can be distinguished and need to be integrated with the clinical evaluation for each patient.

1. A ratio below 1: this is mainly observed in hematoma, myelolipoma, cysts, cavernous angioma, and metastasis of some lower grade renal cancer (even several years after initial surgery of the primary).

2. A moderate tumor uptake (ratio often between 1 and 1.5): adrenocortical adenoma, metastases (renal), pheochromocytoma (sporadic), adrenal hyperplasia, ganglioneuroma, and low-grade ACC.

3. A high tumor uptake (ratio $>3$ ): 5 main diagnoses should be considered: ACC, oncocytic neoplasm (benign or malignant), lymphoma, PHEO (mostly related to SDHB and SDHD), and metastasis. Certain imaging findings can be useful such as heterogeneous uptake with extension into the inferior vena cava for ACC (Fig. 5; Supplemental Fig. 1 [supplemental materials are available at http://jnm.snmjournals.org]), homogeneous and round appearance for oncocytic neoplasms, homogeneous with irregular margins, infiltrative appearance, and potential bilateral adrenal or nodal involvement of lymphoma. The PHEO tumors seen in this setting are almost always secreting and often associated with brown adipose tissue (BAT) uptake mostly due to norepinephrine secretion (34). In the presence of extensive brown adipose tissue activation (especially in periadrenal areas) with a heterogeneous tumor uptake (central photopenic area), it is crucial to check that the assessment of metanephrines has been performed.

Metastases and primary tumor can be detected simultaneously on ${ }^{18}$ F-FDG PET/CT. High-resolution CT chest images should be analyzed carefully to depict a potential primary lung cancer.

Beyond the use of $\mathrm{SUV}_{\max }$ ratio cutoff values, an alternative approach would be to build a predictive model based on metabolic indices and tumor sizes obtained from ${ }^{18} \mathrm{~F}$-FDG PET/CT and calculate an individual risk for malignancy in a given patient.

An adrenal biopsy should only be performed if the results are likely to alter the patient's management and after a PHEO is excluded biochemically, due to the risk of a catecholamineinduced (released by mass biopsy or anesthetics) hypertensive crisis and tachyarrhythmia.

In some centers ${ }^{11} \mathrm{C}$-metomidate-PET/CT is available for characterization of whether the adrenal tumor is of adrenocortical origin, because of the specific tracer uptake in tumors derived from the adrenal cortex. Retroperitoneal sarcomas and other nonadrenocortical tumors are completely ${ }^{11} \mathrm{C}$-metomidate-negative. In the event of a large atypical adrenocortical adenoma, the differentiation between this and an ACC is generally not difficult, because of the irregular ${ }^{11} \mathrm{C}$-metomidate uptake in an atypical adrenocortical adenoma and ability to visualize ACC metastases.

Nonfunctioning Indeterminate Adrenal Masses in Oncologic Patients: ${ }^{18}$ F-FDG PET/CT Is Recommended. Less than $30 \%$ of adrenal masses in patients with a known extraadrenal cancer are related to metastatic disease (12).

The most common primary neoplasms are non-small cell lung carcinoma, lymphoma, colorectal cancer, and renal cell carcinoma. Once an adrenal lesion has been detected in patients with cancer, its characterization is critical to stage the primary disease. PET/CT 
is a highly accurate method for differentiating benign from adrenal metastasis, provided that the primary tumor is itself ${ }^{18} \mathrm{~F}-\mathrm{FDG}$-avid. Despite excellent sensitivity, ${ }^{18} \mathrm{~F}-\mathrm{FDG}$ PET/CT false-negative results can occur with small metastases $<5 \mathrm{~mm}$, hemorrhagic or necrotic tumors, and in tumors with low ${ }^{18} \mathrm{~F}-\mathrm{FDG}$ avidity after chemotherapy. False-negative PET results have been reported in patients with adrenal metastases secondary to pulmonary neuroendocrine tumors, lepidic "bronchioloalveolar" lung cancer, and renal cell carcinoma. False-positive results are reported in inflammatory and infectious processes, PHEO, and, notably, benign adrenal adenoma and hyperplasia. A tumor uptake higher than or equal to that of the liver on visual analysis has an excellent clinical value, with a mean sensitivity and specificity of $97 \%$ and $91 \%$, respectively (35). The published studies, however, have several limitations. They are almost all retrospective, histologic proof (biopsy, surgery, or autopsy) is available for a minority of patients (20\%), and the criterion for malignancy is defined by an increase in size or by tumor regression during chemotherapy. The role of ${ }^{18} \mathrm{~F}$-FDG PET/CT is primarily to establish the extent of metastatic disease, whether the adrenal tumor represents a solitary metastasis or is part of an oligometastatic disease, available for resection or local treatment, or requires systemic therapy. Image-guided adrenal biopsies can be performed safely when noninvasive adrenal imaging tests are inconclusive and after biochemically excluding a PHEO.

\section{CONCLUSION}

Most patients with adrenal tumors present with incidentalomas, and only a minority present with symptoms of adrenal disease. Characterization of these tumors is mostly managed by CT/MRI and biochemistry for most patients. However, for the remaining, more complicated clinical scenarios, nuclear medicine imaging is usually advantageous for establishing an accurate diagnosis. Therefore, an understanding of the radiotracers and their mechanisms for tumor visualization and characterization is important.

\section{DISCLOSURE}

No potential conflict of interest relevant to this article was reported.

\section{KEY POINTS}

QUESTION: Should molecular imaging be considered for assessing an adrenal mass in all patients?

PERTINENT FINDINGS: The first step relies on clinical, biochemi$\mathrm{cal}$, and radiological analysis. Molecular imaging can complement these investigations in certain situations (e.g., disease subtyping in some cases with overt endocrine disease, characterization of indeterminate masses, staging of large tumors).

IMPLICATIONS FOR PATIENT CARE: The evaluation of patients with adrenal mass(es) follows a stepwise approach. Complex cases should be referred to high-volume, specialized centers in order to ensure favorable outcomes.

\section{REFERENCES}

1. Young WF Jr. Clinical practice: the incidentally discovered adrenal mass. $N$ Engl J Med. 2007;356:601-610.

2. Dinnes J, Bancos I, Ferrante di Ruffano L, et al. Management of endocrine disease: imaging for the diagnosis of malignancy in incidentally discovered adrenal masses: a systematic review and meta-analysis. Eur J Endocrinol. 2016;175:R51-R64.
3. Boland GW, Lee MJ, Gazelle GS, Halpern EF, McNicholas MM, Mueller PR. Characterization of adrenal masses using unenhanced CT: an analysis of the CT literature. AJR. 1998;171:201-204.

4. Mayo-Smith WW, Lee MJ, McNicholas MM, Hahn PF, Boland GW, Saini S. Characterization of adrenal masses $(<5 \mathrm{~cm})$ by use of chemical shift MR imaging: observer performance versus quantitative measures. AJR. 1995;165:91-95.

5. Warda MH, Shehata SM, Zaiton F. Chemical-shift MRI versus washout CT for characterizing adrenal incidentalomas. Clin Imaging. 2016;40:780-787.

6. Elsayes KM, Elmohr MM, Javadi S, et al. Mimics, pitfalls, and misdiagnoses of adrenal masses on CT and MRI. Abdom Radiol (NY). 2020;45:982-1000.

7. Sundin A. Imaging of adrenal masses with emphasis on adrenocortical tumors. Theranostics. 2012;2:516-522.

8. Mayo-Smith WW, Song JH, Boland GL, et al. Management of incidental adrenal masses: a white paper of the ACR Incidental Findings Committee. J Am Coll Radiol. 2017;14:1038-1044.

9. Fassnacht M, Arlt W, Bancos I, et al. Management of adrenal incidentalomas: European Society of Endocrinology Clinical Practice Guideline in collaboration with the European Network for the Study of Adrenal Tumors. Eur J Endocrinol. 2016;175: G1-G34.

10. Marty M, Gaye D, Perez P, et al. Diagnostic accuracy of computed tomography to identify adenomas among adrenal incidentalomas in an endocrinological population. Eur J Endocrinol. 2018;178:439-446.

11. Woo S, Suh CH, Kim SY, Cho JY, Kim SH. Pheochromocytoma as a frequent falsepositive in adrenal washout CT: A systematic review and meta-analysis. Eur Radiol. 2018;28:1027-1036.

12. Hammarstedt L, Muth A, Sigurjonsdottir HA, et al. Adrenal lesions in patients with extra-adrenal malignancy: benign or malignant? Acta Oncol. 2012; 51:215-221.

13. Bourdeau I, Parisien-La Salle S, Lacroix A. Adrenocortical hyperplasia: A multifaceted disease. Best Pract Res Clin Endocrinol Metab. 2020;34:101386.

14. Khan TS, Sundin A, Juhlin C, Langstrom B, Bergstrom M, Eriksson B. ${ }^{11}$ C-metomidate PET imaging of adrenocortical cancer. Eur J Nucl Med Mol Imaging. 2003;30: $403-410$.

15. Vezzosi D, Tenenbaum F, Cazabat L, et al. Hormonal, radiological, NP-59 scintigraphy, and pathological correlations in patients with Cushing's syndrome due to primary pigmented nodular adrenocortical disease (PPNAD). J Clin Endocrinol Metab. 2015; 100:4332-4338.

16. Louiset E, Duparc C, Young J, et al. Intraadrenal corticotropin in bilateral macronodular adrenal hyperplasia. N Engl J Med. 2013;369:2115-2125.

17. Funder JW, Carey RM, Mantero F, et al. The management of primary aldosteronism: case detection, diagnosis, and treatment: an Endocrine Society Clinical Practice Guideline. J Clin Endocrinol Metab. 2016;101:1889-1916.

18. Dekkers T, Prejbisz A, Kool LJS, et al. Adrenal vein sampling versus CT scan to determine treatment in primary aldosteronism: an outcome-based randomised diagnostic trial. Lancet Diabetes Endocrinol. 2016;4:739-746.

19. Beuschlein F, Mulatero P, Asbach E, et al. The SPARTACUS Trial: controversies and unresolved issues. Horm Metab Res. 2017;49:936-942.

20. Wong KK, Fig LM, Youssef E, Ferretti A, Rubello D, Gross MD. Endocrine scintigraphy with hybrid SPECT/CT. Endocr Rev. 2014;35:717-746.

21. Bergström M, Juhlin C, Bonasera TA, et al. PET imaging of adrenal cortical tumors with the 11 beta-hydroxylase tracer ${ }^{11} \mathrm{C}$-metomidate. J Nucl Med. 2000;41: 275-282.

22. Burton TJ, Mackenzie IS, Balan K, et al. Evaluation of the sensitivity and specificity of ${ }^{11} \mathrm{C}$-metomidate positron emission tomography (PET)-CT for lateralizing aldosterone secretion by Conn's adenomas. J Clin Endocrinol Metab. 2012;97: 100-109.

23. Hahner S, Kreissl MC, Fassnacht M, et al. Functional characterization of adrenal lesions using [ ${ }^{123}$ I]IMTO-SPECT/CT. J Clin Endocrinol Metab. 2013;98:15081518 .

24. Heinze B, Fuss CT, Mulatero P, et al. Targeting CXCR4 (CXC chemokine receptor type 4) for molecular imaging of aldosterone-producing adenoma. Hypertension. 2018;71:317-325.

25. Ding J, Zhang Y, Wen J, et al. Imaging CXCR4 expression in patients with suspected primary hyperaldosteronism. Eur J Nucl Med Mol Imaging. 2020;47: 2656-2665.

26. Taïeb D, Jha A, Guerin C, et al. ${ }^{18}$ F-FDOPA PET/CT Imaging of MAX-related pheochromocytoma. J Clin Endocrinol Metab. 2018;103:1574-1582.

27. Taïeb D, Hicks RJ, Hindie E, et al. European Association of Nuclear Medicine Practice Guideline/Society of Nuclear Medicine and Molecular Imaging Procedure Standard 2019 for radionuclide imaging of phaeochromocytoma and paraganglioma. Eur J Nucl Med Mol Imaging. 2019;46:2112-2137.

28. Kan Y, Zhang S, Wang W, Liu J, Yang J, Wang Z. ${ }^{68}$ Ga-somatostatin receptor analogs and ${ }^{18}$ F-FDG PET/CT in the localization of metastatic pheochromocytomas and 
paragangliomas with germline mutations: a meta-analysis. Acta Radiol. 2018;59: 1466-1474.

29. Han S, Suh CH, Woo S, Kim YJ, Lee JJ. Performance of ${ }^{68}$ Ga-DOTA-conjugated somatostatin receptor-targeting peptide PET in detection of pheochromocytoma and paraganglioma: a systematic review and metaanalysis. J Nucl Med. 2019;60: 369-376.

30. Yamamoto S, Hellman P, Wassberg C, Sundin A. ${ }^{11}$ C-hydroxyephedrine positron emission tomography imaging of pheochromocytoma: a single center experience over 11 years. J Clin Endocrinol Metab. 2012;97:2423-2432.

31. Vyakaranam AR, Crona J, Norlen O, Hellman P, Sundin A. ${ }^{11} \mathrm{C}$-hydroxy-ephedrine$\mathrm{PET} / \mathrm{CT}$ in the diagnosis of pheochromocytoma and paraganglioma. Cancers (Basel). $2019 ; 11$.
32. Kim SJ, Lee SW, Pak K, Kim IJ, Kim K. Diagnostic accuracy of ${ }^{18}$ F-FDG PET or PET/CT for the characterization of adrenal masses: a systematic review and metaanalysis. Br J Radiol. 2018;91:20170520.

33. Guerin C, Pattou F, Brunaud L, et al. Performance of ${ }^{18}$ F-FDG PET/CT in the characterization of adrenal masses in noncancer patients: a prospective study. JClin Endocrinol Metab. 2017;102:2465-2472.

34. Abdul Sater Z, Jha A, Hamimi A, et al. Pheochromocytoma and paraganglioma patients with poor survival often show brown adipose tissue activation. $J$ Clin Endocrinol Metab. 2020;105:1176-1185.

35. Boland GW, Dwamena BA, Jagtiani Sangwaiya M, et al. Characterization of adrenal masses by using FDG PET: a systematic review and meta-analysis of diagnostic test performance. Radiology. 2011;259:117-126. 